
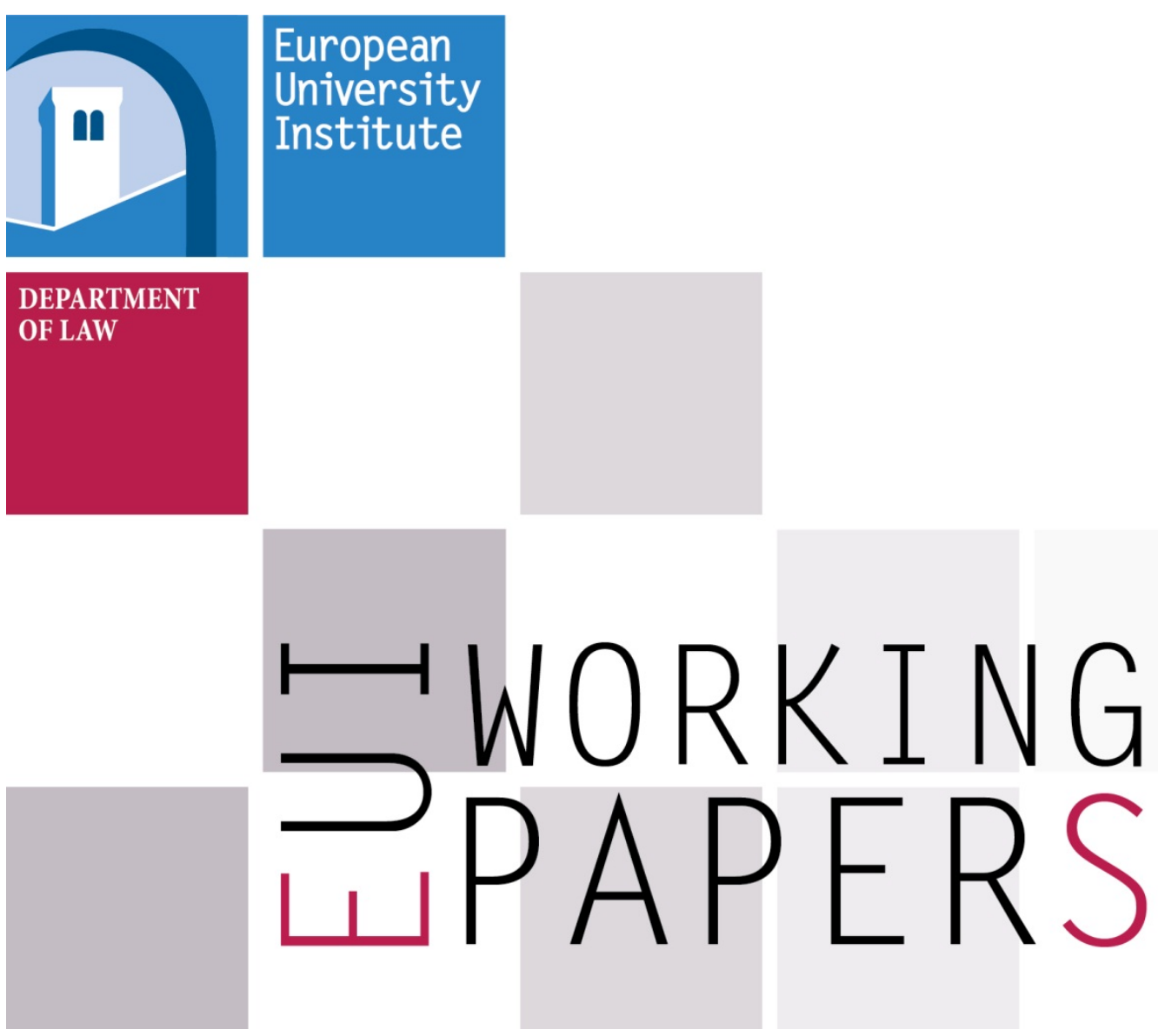

LAW 2015/44

Department of Law

L'esercizio extraterritoriale della giurisdizione civile con riferimento alle gravi violazioni dei diritti umani da parte delle imprese multinazionali

Claire Bright 

European University Institute

Department of Law

\section{L'ESERCIZIO EXTRATERRITORIALE DELLA GIURISDIZIONE CIVILE CON RIFERIMENTO ALLE GRAVI VIOLAZIONI DEI DIRITTI UMANI}

Claire Bright

EUI Working Paper LAW 2015/44 
This text may be downloaded for personal research purposes only. Any additional reproduction for other purposes, whether in hard copy or electronically, requires the consent of the author. If cited or quoted, reference should be made to the full name of the author, the title, the working paper or other series, the year, and the publisher.

ISSN 1725-6739

(C) Claire Bright, 2015

Printed in Italy

European University Institute

Badia Fiesolana

I-50014 San Domenico di Fiesole (FI)

Italy

www.eui.eu

cadmus.eui.eu 
Author Contact Details

Claire Bright

claire.bright@eui.eu 


\begin{abstract}
Il diritto internazionale privato può giocare un ruolo chiave nel regolare le imprese multinazionali, garantendo allo stesso tempo un accesso effettivo alla giustizia per le vittime. In particolare, diversi Stati hanno adottato meccanismi giurisdizionali che prevedono l'esercizio della giurisdizione extraterritoriale civile da parte delle loro corte per reprimere le violazioni dei diritti umani commesse da imprese multinazionali domiciliate oppure semplicemente 'presente' nel loro territorio. Tali meccanismi sono particolarmente rilevanti quando lo Stato ospitante è incapace o riluttante ad assolvere all'obbligo di proteggere i diritti umani delle persone che si trovano sul suo territorio, anche qualora gli abusi siano commessi da persone giuridiche, e di rimediare a tali abusi quando accadono.

Con questo studio, si intende valutare la legittimità di tal esercizio extraterritoriale della giurisdizione civile con riferimento alle gravi violazioni dei diritti umani commesse da imprese multinazionali in Paesi in via di sviluppo alla luce del diritto internazionale. In quest'ottica, occorre distinguere la situazione della giurisdizione esercitata da parte dello Stato di origine dell'impresa multinazionale da quella di uno Stato terzo sul cui territorio l'impresa è semplicemente 'presente'.
\end{abstract}

\title{
Keywords
}

Diritto internazionale privato, esercizio extraterritoriale della giurisdizione, imprese multinazionali, accesso alla giustizia, diritti umani 


\section{Table of contents}

INTRODUCTION

1

L'ESERCIZIO EXTRATERRITORIALE DI GIURISDIZIONE CIVILE DA PARTE DELLO STATO

DI ORIGINE DELL'IMPRESA MULTINAZIONALE

L'ESERCIZIO DELLA GIURISDIZIONE UNIVERSALE NEL CAMPO CIVILE DA PARTE DI UN PAESE DOVE L'IMPRESA È SEMPLICEMENTE 'PRESENTE'

CONCLUSIONE. . .9 



\section{Introduction}

L'ultimo secolo ha visto un aumento vertiginoso del numero delle imprese multinazionali ${ }^{1}$ che è passato in una cinquantina di anni da circa 7000 a più di $80000 .^{2}$ Nello stesso tempo, il loro peso economico è aumentato notevolmente, ${ }^{3}$ cosicché buona parte delle prime cento potenze economiche mondiali è ormai costituita da imprese multinazionali. ${ }^{4}$ Parallelamente a questo potenziamento economico, le imprese multinazionali si sono anche rivelate in grado di svolgere un'influenza decisiva sul piano politico. L'impresa multinazionale ha acquisito una tale posizione egemonica sotto la spinta della globalizzazione economica, e, più particolarmente, per via dell'abbassamento dei costi dei trasporti e delle comunicazioni, della liberalizzazione del commercio internazionale, e della riduzione delle restrizioni agli investimenti diretti esteri. ${ }^{5}$ Nella ricerca di una competitività sempre maggiore, l'impresa multinazionale tende a spostare parte della sua attività produttiva ricercando la localizzazione ottimale per ogni frazione della sua produzione in funzione dei vantaggi comparati offerti da ogni Paese. In questo modo, può sfruttare, inoltre ai fattori di produzione a basso costo e le risorse naturali abbondanti, i vantaggi offerti dalle differenze tra ordinamenti giuridici, in particolare in materia di normativa fiscale, sociale ed ambientale, che incidono sui costi di produzione. L'unità economica dell'impresa multinazionale è completamente ignorata dal diritto, che si rifiuta di considerare il gruppo internazionale di società nel suo insieme. ${ }^{6}$ Di conseguenza, ogni ente dell'impresa, dotato di autonoma personalità giuridica, è normalmente sottoposto al sistema giuridico dello Stato ospitante, cioè dello Stato territoriale ove opera, che è spesso un Paese in via di sviluppo. ${ }^{7}$ Il problema è che la combinazione tra la maggiore permissività delle legislazioni dei Paesi ospitanti, da un lato, la mancanza di vincolatività delle norme internazionali nei confronti delle imprese multinazionali, dall'altro, si traduce in pratica in un'impunità totale per gli abusi commessi nel condurre le loro attività nei Paesi in via di sviluppo, anche in relazione alle violazioni dei diritti umani. $^{8}$ Infatti, spesso, gli apparati e i sistemi giudiziari dei Paesi ospitanti non sono in grado di garantire adeguatamente la tutela dei diritti umani, o di offrire alle vittime un accesso effettivo alla giustizia. ${ }^{9}$ Oltre ai difetti dei sistemi giuridici, si unisce spesso una mancanza di volontà da parte dei

L'autore è Senior Lecturer alla London School of Business and Management e Programme Associate nel Research Programme on Civil Justice Systems del Centre for Socio-Legal Studies dell'Università di Oxford. L'autore vorrebbe ringraziare Maria-Grazia Porcedda e Maddalena Neglia per i loro commenti in merito a questo studio.

${ }^{1}$ Si veda a proposito della definizione di imprese multinazionali, P.T. Muchlinski, Multinational Enterprises and the Law (Blackwell, 1999), at 12-15. Si veda anche F. Francioni, Imprese multinazionali, Protezione diplomatica e responsabilità internazionale (Dott. A. Giuffrè, 1979) at 8 , che sottolinea che: "la 'multinazionalità' è un attibuto puramente di fatto essendo esse nient'altro che gruppi di società dislocate e operanti in una pluralità di Stati e dotate, ognuna, di diversa nazionalità in corrispondenza della Stato territoriale ove operano o con cui, comunque, sussistono i collegamenti idonei ai fini dell'attribuzione della nazionalità medesima".

${ }^{2}$ M.-A. Hennerbert, 'L'essor historique des entreprises multinationales - Comprendre l'émergence et la montée en puissance du nouveau 'Léviathan' des temps modernes" (inverno 2007), Dire 46.

${ }^{3}$ A. Chandler, 'The Beginning of Big Business in American Industry', (1959) 33 Business History Review 79.

${ }^{4}$ United Nations Conference on Trade and Development (UNCTAD), World Investment Report 2009: 'Transnational Corporations, Agricultural Production and Development', 223.

${ }^{5}$ United Nations, World Investment Report, New York and Geneva, United Nations, 2004.

${ }^{6}$ G. Peroni e C. Migani, 'La responsabilità sociale dell'impresa multinazionale nell'attuale contesto internazionale' (2010) Ianus, 2 International Journal of Law and Finance 1.

${ }^{7}$ F. Francioni, op. cit. supra nota 1 at 15: "L'IMN è caratterizzata dalla dicotomia fra 'unità' economica del gruppo e 'diversità' giuridica delle varie unità operanti nei diversi Stati di cui assumono la nazionalità ed ai cui ordinamenti interni esse soggiacciono sia per quanto concerne la loro origine e costituzione, sia per quanto concerne la disciplina delle relative attività".

${ }^{8}$ R. Caloia, Imprese multinazionali e responsabilità sociale: limiti ed opportunità alla luce del Diritto Internazionale attuale (Tesi di Dottorato, Università degli studi di Napoli Federico II), at 133.

${ }^{9}$ A. Bonfanti, 'Diritti umani e imprese multinazionali dinanzi ai giudici europei: sulla revisione del 
governi di questi paesi nel regolare le imprese multinazionali per paura di rendersi meno attraenti per gli investimenti esteri, cruciali per la loro crescita economica. ${ }^{10}$ I numerosi casi di gravi violazioni dei diritti umani nei quali sono coinvolte in maniera diretta o indiretta delle imprese multinazionali stano a testimoniare la necessità di una maggiore regolamentazione delle imprese multinazionali relativamente alle loro attività nei paesi in via di sviluppo.

In quest'ottica, i meccanismi giurisdizionali di diritto internazionale privato possono giocare un ruolo chiave nel regolare le imprese multinazionali, garantendo allo stesso tempo un accesso effettivo alla giustizia per le vittime. In particolare, diversi Stati hanno adottato meccanismi giurisdizionali che prevedono l'esercizio della giurisdizione extraterritoriale civile da parte dei loro tribunali nazionali per reprimere le violazioni dei diritti umani commesse da imprese multinazionali domiciliate oppure semplicemente 'presenti' nel loro territorio. Tali meccanismi sono particolarmente rilevanti quando lo Stato ospitante è incapace o riluttante ad assolvere all'obbligo di proteggere i diritti umani delle persone che si trovano sul suo territorio, anche qualora gli abusi siano commessi da persone giuridiche, e di rimediare a tali abusi quando accadono.

\section{L'esercizio extraterritoriale di giurisdizione civile da parte dello Stato di origine dell'impresa multinazionale}

Diversi ordinamenti giuridici hanno adottato meccanismi giurisdizionali che prevedono l'esercizio della giurisdizione civile extraterritoriale delle loro corti per reprimere le violazioni dei diritti umani commesse da imprese multinazionali domiciliate nel loro territorio. Nell'ambito dell'Unione Europea, il Regolamento (UE) n. 1215/2012 del Parlamento Europeo e del Consiglio del 12 dicembre 2012 ("Bruxelles I bis") 11 attribuisce competenza giurisdizionale ai tribunali degli Stati membri per accogliere azioni civili promosse contro convenuti domiciliati nel loro territorio, a prescindere dalla nazionalità delle parti, il luogo di residenza dell'attore, oppure il luogo dove il reato presunto si sia verificato. ${ }^{12}$ Sebbene non ci sia nel diritto europeo alcun criterio giurisdizionale specifico per le azioni

(Contd.)

Regolamento (CE) n. 44/2001', (2011) 3 Rivista di Diritto Internazionale Privato e Processuale 697, at 716: "Talvolta accade infatti che tali Paesi non siano dotati di apparati e sistemi giudiziari efficenti, o che non permettano la presentazione di class actions, con conseguente aumento dei costi gravanti sugli attori."; P. Henner, Human Rights and the Alien Tort Statute: Law, History and Analysis, (American Bar Association, 2009) 4: "In an ideal world, all disputes could be handled either within the borders of the country where the dispute arises, or, if the dispute involves residents of different nations, in some form of international tribunal (...) However, we do not live in an ideal world. The judicial systems of many countries are underdeveloped and, in some cases, are either corrupt or under the inflence, it not control, of authoritarian regimes;" M. Winkler, Imprese multinazionali e diritto internazionale nell'era della globalizzazione (Milano: Giuffré, 2008) 256.

${ }^{10}$ U. Musumeci, 'L'impatto sociale delle mutiazionali', in L. Sacconi (ed.), Guida critica alla Responsabilità sociale d'impresa e al governo d'impresa (Bancaria editrice, 2005) 561.

${ }^{11}$ Regolamento (UE) n. 1215/2012 del Parlamento Europeo e del Consiglio del 12 dicembre 2012 ("Bruxelles I bis") concernente la competenza giurisdizionale. Il Regolamento si applica a decorrere dal 10 gennaio 2015 ad eccezione degli articoli 75 e 76 che si applicano a decorrere dal 10 gennaio 2014 e abroga il regolamento (CE) n.44/2001 del Consiglio del 22 dicembre 2000 concernente la competenza giurisdizionale, il riconoscimento e l'esecuzione delle decisioni in materia civile e commerciale, che ha sostituito la Convenzione di Bruxelles del 1968 concernente la competenza giurisdizionale e l'esecuzione delle decisioni in materia civile e commerciale.

${ }^{12}$ L'articolo 4.1. del Regolamento Bruxelles I bis prevede che: "A norma del presente regolamento, le persone domiciliate nel territorio di un determinato Stato membro sono convenute, a prescindere dalla loro cittadinanza, davanti alle autorità giurisdizionali di tale Stato membro." Inoltre a questo criterio giurisdizionale, altri criteri stabiliti nel Regolamento permettono di attrare l'imprese domiciliata sul territorio di uno Stato membro in un altro Stato membro. In particolare, in materia di illeciti civili dolosi o colposi, l'articolo 7.2 del Regolamento Bruxelles I bis permette di attrare un convenuto domiciliato sul territorio di uno Stato membro davanti ai giudici del luogo in cui l'evento dannoso è avvenuto o può avvenire. Quest'ultima espressione è stata interpretata dalla giurisprudenza della Corte di Giustizia delle Comunità Europee come lasciando una scelta all'attore tra il luogo in cui il danno si sia verificato e il luogo in cui l'atto che ha dato origine al danno sia avvenuto quando essi non correspondono. Per quanto riguarda le azioni civili contro imprese multinazionali domiciliate nell'Unione Europea per abusi comessi al di fuori del territorio europeo, tale criterio 
di risarcimento dei danni nei confronti alle violazioni dei diritti umani da parte d'imprese multinazionali, il Parlamento europeo ha tenuto a precisare che il criterio giurisdizionale del domicilio del convenuto può servire da base giuridica per stabilire la competenza dei tribunali degli Stati membri dell'Unione per le controversie relative a violazioni dei diritti umani compiute in paesi terzi da imprese multinazionali domiciliate sul territorio dell'Unione europea. ${ }^{13}$ L'articolo 63 stabilisce a questo riguardo che "una società o altra persona giuridica è domiciliata nel luogo in cui si trova a) la sua sede statutaria; b) la sua amministrazione centrale: oppure c) il suo centro d'attività principale." ${ }^{14}$ Si tratta, indubbiamente, di una regola che favorisce l'accesso ai giudici europei per gli attori poiché è sufficiente che l'impresa abbia sul territorio di uno Stato membro la sua sede statutaria, la sua amministrazione centrale, oppure il suo centro d'attività principale per fondare la competenza del foro di questo Stato. Diversi Stati membri hanno esercitato la loro giurisdizione extraterritoriale su tale fondamento. In particolare, in base alla localizzazione in Inghilterra del domicilio delle società madri d'imprese multinazionali, diverse azioni civili sono state instaurate dinanzi alle corti inglesi per i gravi danni alla salute subiti dalle vittime in consegenza del lavoro svolto presso le loro affiliate in Africa nei settori dell'estrazione dell'uranio ${ }^{15}$ dell'amianto, ${ }^{16}$ oppure del trattamento del mercurio. ${ }^{17}$ I tribunali inglesi hanno rifiutato di declinare la propria giurisdizione a favore dei giudici africani sulla base del forum non conveniens (che all'epoca non era stato escluso della Corte di giustizia dell'Unione europea nello spazio giudiziario europeo ${ }^{18}$ ) ritenendo che l'accesso efficace alla giustizia non sarebbe stato garantito se il processo si fosse svolto dinanzi ai giudici africani. Tali ricorsi erano diretti alle società madri inglesi chiamate a rispondere dei danni posti in essere dalle sussidiarie in merito alla mancata osservanza del loro obbligo di diligenza nella prevenzione dei rischi ben conosciuti dell'esposizione a tali sostanze. ${ }^{19}$ Più recentemente, la società madre inglese di un'impresa multinazionale è stata citata in giudizio a seguito di una class action di circa 30000 cittadini africani instaurata dinanzi alle corti inglesi, per responsabilità diretta (e non indiretta, questa volta) dei danni subiti dalla popolazione

\section{(Contd.)}

giurisdizionale permette all'attore di attrare l'imprese dinanzi ai corti di un altro Stato membro nella situazione nella quale una decisione presa in uno Stato diverso dello Stato dov'è l'imprese è domiciliata abbia arrecato il danno. Inoltre, secondo l'articolo 7.5 del Regolamento Bruxelles I bis, una persona domiciliata in uno Stato membro può essere convenuta in un altro Stato membro "qualora si tratti di controversia concernente l'esercizio di una succursale, di un'agenzia o di qualsiasi altra sede d'attività, davanti all'autorità giurisdizionale del luogo in cui essa è situata". Si deduce dunque che, nella situazione nella quale il danno sia stato caggionato da una succursale, un'agenzia o qualsiasi altra sede d'attività dell'impresa, l'azione potrà essere perseguita nello Stato in cui quest'ultima opera. Si veda R. Caloia, op. cit. supra nota 8 , at 117.

${ }^{13}$ Risoluzione sul Libro Verde della Commissione, "Promuovere un quadro europeo per la responsabilità sociale delle imprese", COM (2001) 366

${ }^{14}$ L'articolo 63.2 aggiunge che: "Per quanto riguarda l'Irlanda, Cipro e il Regno Unito, per 'sede statutaria' si intende il 'registered office' o, se non esiste alcun 'registered office', il 'place of incorporation' (luogo di acquisizione della personalità giuridica), ovvero, se nemmeno siffatto luogo esiste, il luogo in conformità della cui legge è avvenuta la «formation» (costituzione).

${ }^{15}$ Connelly v RTZ Corporation (1998) AC 854 (HL). Si veda R. Meeran, Liability of Multinational Corporations: A Critical Stage in the UK, in Kamminga et Zia-Zarifi (eds.), Liability of Multinational Corporations under International Law, The Hague 2000, p. 258.

${ }^{16}$ Adams v Cape Industries (1990) Ch 433 (CA); Lubbe v Cape Plc (2000) 4 All ER 268 (HL). R. Meeran, 'Cape P1: South African Mineworkers' Quest for Justice' (2003) 9 International Journal of Occupational Environmental Health 218; P. Mulchlinski, 'Coporations in International Litigation: Problems of Jurisdiction and the United Kingdom Asbestos Cases' (2001) International and Comparative Law Quarterly 1.

${ }^{17}$ Ngcobo $v$ Thor Chemicals Holdings Ltd The Times 10 Novembre 1995 (CA); Sithole $v$ Thor Chemicals Holdings Ltd and Another (1999) All ER (D) 102 (CA). Si veda, Oxford Pro Bono Publico, 'Obstacles to Justice and Redress for Victims of Corporate Human Rights Abuse - A Comparative Submission Prepared for Prof. John Ruggie, UN SG Special Representative on Business and Human Rights', 3 Novembre 2008, www.law.ox.ac.uk/opbp p. 270; R. Meeran, 'Tort Litigation against Multinational Corporations for Violation of Human Rights: An Overiew of the Position Outside the United States', (2011) 3 City University of Hong Kong Law Review 25.

${ }^{18}$ CJUE, 1 marzo 2005, Owusu c. Jackson, C-281/02

${ }^{19}$ R. Caloia, op. cit. supra nota 8, at 123. 
locale in seguito allo scarico di rifiuti tossici in Costa d'Avorio realizzato dalla propria sussidiaria africana sulle sue istruzioni.

Sebbene la maggior parte di questi casi si sia risolta con un accordo stragiudiziale, hanno permesso alle vittime di ottenere un effettivo risarcimento per i danni subiti. Essi sono dunque rilevante in quanto confermano il ruolo cruciale dell'esercizio extraterritoriale della giurisdizione civile degli Stati membri per garantire l'accesso efficace alla giustizia per le vittime di abusi che le imprese multinazionali commettono nei paesi terzi.

Per quanto riguarda la legittimità di tale esercizio extraterritoriale della giurisdizione civile alla luce del diritto internazionale, bisogna esaminare i principali titoli di giurisdizione largamente utilizzati ed accettati in diritto internazionale, che sono in quattro: il principio di territorialità, il principio di personalità, il principio di protezione e il principio di universalità. Tra questi principi, il principio di personalità attiva permette allo Stato di origine dell'impresa di esercitare la giurisdizione extraterritoriale allo scopo di regolare la condotta de propri nazionali - persone fisiche o giuridiche anche al di fuori del suo territorio nazionale. Tale principio è stato a volte esteso per includere anche le persone che, pur non avendo la nazionalità dello Stato in questione, avevano, sul suo territorio, la loro residenza abituale. Di conseguenza, applicato alle imprese multinazionali, il principio della personalità attiva legittima la giurisdizione dello Stato del quale la società madre ha la nazionalità o ove possiede il suo domicilio per regolare le attività che sviluppano anche all'estero. O. De Schutter ha sottolineato che il ricorso al principio di personalità come base giuridica all'esercizio della giurisdizione extraterritoriale è particolarmente ben adattato nei confronti delle imprese multinazionali, permettendo alla società madre di controllare gli enti sui quali può esercitare un'influenza. ${ }^{20}$ Due giustificazioni in particolare servono tradizionalmente da fondamento all'esercizio della giurisdizione extraterritoriale sulla base del principio di personalità per regolare la condotta di persone fisiche, che si applicano ugualmente alle persone giuridiche. In primo luogo, in assenza di estradizione di presunti autori di violazioni del diritto internazionale, l'esercizio extraterritoriale della giurisdizione assicurerebbe che certi crimini non rimangano impuniti. Poiché le persone giuridiche non possono essere estradate, l'esercizio extraterritoriale della giurisdizione da parte dello Stato di origine è particolarmente importante per assicurare che l'impresa sarà perseguita in caso di abusi. In secondo luogo, l'esercizio della giurisdizione extraterritoriale servirebbe allo Stato di origine per assicurarsi che i suoi cittadini non violino determinati valori fondamentali quando operino all'estero, o per reprimere tali violazioni. In materia di tutela dei diritti umani, l'esercizio extraterritoriale della giurisdizione da parte dello Stato di origine permette di contribuire al loro rispetto, nel Paese dove l'impresa multinazionale opera. In particolare per quanto riguarda i diritti umani universalmente riconosciuti, tale esercizio della giurisdizione extraterritoriale potrebbe difficilmente essere considerato un'ingerenza negli affari degli altri Stati giacché il loro rispetto sarebbe nell'interesse dell'intera comunità internazionale. ${ }^{21}$

Permesso dai principi generali del diritto internazionale, l'esercizio extraterritoriale della giurisdizione civile da parte dello Stato di origine dell'impresa potrebbe essere considerato come reso obbligatorio dall'emergente obbligo in diritto internazionale, in capo a quest'ultimo Stato, di regolare le sue imprese multinazionali, anche quando operano all'estero, al fine di prevenire la commissione di violazioni dei diritti umani e di reprimerle quando succedono. ${ }^{22}$

\footnotetext{
${ }^{20}$ O. De Schutter, 'Extraterritorial Jurisdiction as a tool for improving the Human Rights Accountability of Transnational Corporations', (2006) Faculté de Droit de l'Université Catholique de Louvain 24.

${ }^{21}$ R. Caloia, op. cit. supra nota 8, at 144.

${ }^{22} \mathrm{Si}$ veda C. Bright, L'accès à la justice civile en cas de violations des droits de l'homme par des entreprises multinationales (Florence: European University Institute, Cadmus, 2013).
} 


\section{L'esercizio della giurisdizione universale nel campo civile da parte di un Paese dove l'impresa è semplicemente 'presente'}

Nel campo civile, il concetto di giurisdizione universale si riferisce all'esercizio della propria giurisdizione da parte di tribunali nazionali per perseguire il presunto autore di un reato considerato di natura tale da offendere la comunità internazionale nel suo complesso. Tal tipo di giurisdizione è stato esercitato, in modo controverso, ${ }^{23}$ dalle corti americane in base all'Alien Tort Statute ("ATS"). L'ATS è uno statuto federale che fu adottato dal primo Congresso statunitense nel 1789 e che conferisce ai tribunali federali statunitensi competenza giurisdizionale per cause civili instaurate da cittadini stranieri per torti subiti in violazione del diritto delle Nazioni (law of nations) o di un trattato internazionale concluso dagli Stati Uniti. ${ }^{24}$ Tale legge, rimasta 'dormiente' per quasi due secoli, ${ }^{25}$ rinacque nel 1980, con il caso Filártiga v. Pena Irala ${ }^{26}$ nel quale la Corte federale di appello per il Secondo distretto ritenne che essa le conferisse giurisdizione relativamente ad un'azione di risarcimento dei danni promossa da cittadini paraguayani (residenti negli Stati Uniti) contro un ex ufficiale paraguayano (in visita negli Stati Uniti) per atti di tortura commessi in Paraguay. La corte affermò la sua giurisdizione ratione materiae in base al fatto che tali atti di tortura violano norme di diritto internazionale dei diritti umani universalmente accettate, qualsiasi fosse la nazionalità delle parti. Inoltre, la corte considerò che il fatto che la citazione fosse stata consegnata personalmente al convenuto in giudizio era sufficiente per soddisfare la condizione procedurale di giurisdizione in personam. Il convenuto fu poi condannato a pagare ai ricorrenti più di 10 milioni di dollari di indennizzo. L'importanza di questo caso deriva del fatto che esso aprì la giurisdizione dei tribunali civili statunitensi a vittime straniere di violazioni dei diritti umani permettendo loro di intraprendere un'azione civile contro presunti autori anche stranieri - semplicemente presenti sul territorio del foro indipendentemente dal luogo in cui il reato fosse stato commesso. Un'interpretazione così audace della legge è stata poi confermata dalla Corte Suprema nella sua sentenza sul caso Sosa v. AlvarezMachain, ${ }^{27}$ sebbene in tale sentenza la corte ha ridotto il numero delle violazioni internazionali atte ad introdurre azione dinanzi ai tribunali federali statunitensi in base all'ATS. In effetti, la corte ha specificato che l'ATS si limitava a stabilire un criterio di giurisdizione a favore delle corti federali ${ }^{28}$ per certi tipi di violazioni gravi del diritto internazionale definiti in norme specifiche, universali e cogenti. ${ }^{29}$ Di conseguenza, solo le violazioni più gravi dei diritti umani che sono oggetto di riprovazione universale possono fondare la competenza delle corti federali statunitensi in base

${ }^{23}$ I. Moulier, 'Observations sur l'Alien Tort Claims Act et ses implications internationales' (2003) 49 Annuaire français de droit international 129 , at 131.

${ }^{24}$ L'Alien Tort Statute (Judiciary Act of 1789, ch. 20, § 9(b), 1 Stat. 73, 77 (1789), codificato in 28 U.S.C. § 1350), recita: "The district courts shall have original jurisdiction of any civil action by an alien for a tort only, committed in violation of the law of nations or a treaty of the United States". B. Stephens, J. Chomsky, J. Green, P. Hoffman, and M. Ratner, International Human Rights Litigation in U.S. Courts (2nd ed., Martinus Nijhoff Publishers, 2008); P. Henner, Human Rights and the Alien Tort Statute: Law, History and Analysis (American Bar Association, 2009).

${ }^{25}$ Durante questi due secoli, una ventina di casi furono instraurati in base all'ATS e soltanto due con successo (Adra v. Clift, 195 F. Supp. 857 (D.Md.1961) e Bolchos v. Darrel, 1 Bee 74, 3 Fed.Cas. 810 (D.S.C.1795)). K.C. Randall, 'Federal Jurisdiction over International Law Claims: Inquiries into the Alien Tort Statute', (1985) 18 NYU Journal of International Law and Politics 4; J. K., Elsea, 'The Alien Tort Statute : Legislative History and Executive Branch Views', (2003) October 2 CRS Report for Congress.

${ }^{26}$ Filártiga v. Peña-Irala, 630 F.2d 876 (2d Cir. 1980).

${ }^{27}$ Sosa v. Alvarez-Machain, 542 U.S. 692, 124 S. Ct. 2739, 159 L. Ed. 2 d 718 (2004).

${ }^{28}$ M. Winkler, 'Le imprese multinazionali e l'oscillante giurisprudenza dell'Alien Tort Statute' (2012) Rivista di diritto processuale internazionale e arbitrato internazionale 40 , at 41 .

${ }^{29}$ Sosa v. Alvarez-Machain, 542 U.S. 692: "courts should require any claim based on the present-day law of nations to rest on a norm of international character accepted by the civilized world and defined with a specificity comparable to the features of the 18th-century paradigms". 
all'ATS ${ }^{30}$ D'ora in poi, le violazioni del diritto internazionale che sono state considerate rispondenti a questo criterio includono genocidio e crimini contro l'umanità, ${ }^{31}$ crimini di guerra, ${ }^{32}$ atti di tortura, ${ }^{33}$ trattamenti crudeli, inumani e degradanti, uccisioni extragiudiziali, ${ }^{34}$ lavoro sforzato, ${ }^{35}$ e sparizione forzata, ${ }^{36}$ generalmente commesse nei Paesi in via di sviluppo. ${ }^{37}$

Il campo di applicazione dell'ATS è stato poi esteso quando la Corte federale di appello per il Secondo distretto ha deciso che l'ATS poteva essere invocato non solamente contro funzionari pubblici ma anche privati (qualora essi agiscano al di fuori della propria funzione ufficiale) che commettano violazioni del diritto internazionale. ${ }^{38}$ Di conseguenza, gli individui ed anche le imprese multinazionali sono stati portati in giudizio in virtù dell'ATS. Da allora, un centinaio di azioni sono state instaurate dinanzi alle corti federali statunitensi sulla base dell'ATS da attori non-americani contro imprese multinazionali sia americane che straniere accusate di aver commesso direttamente, ${ }^{39} \mathrm{o}$ di essersi rese complici ${ }^{40}$ di violazioni dei diritti umani anche qualora queste siano avvenute al di fuori del territorio statunitense. In relazione alle imprese non-americane citate in giudizio, le corti federali si sono accontentate, come criterio di collegamento, del fatto che fossero semplicemente 'presenti' sul territorio del foro, avendovi un ufficio, una rappresentanza oppure avendovi condotto affari in modo stabile e continuativo (doing business). ${ }^{41}$ In un caso, è stato considerato che bastava il fatto che l'impresa avesse un ufficio rappresentativo a New York.

Questa linea di processi basati sull'ATS è stata messa in discussione quando, nel settembre 2010, la Corte federale di appello per il Secondo distretto, nel caso Kiobel v. Royal Dutch Petroleum Co. ${ }^{42}$, ha considerato che lo statuto non si applicava alle persone giuridiche come le imprese multinazionali. Il caso Kiobel $^{43}$ è stato instaurato da un gruppo di abitanti del delta del Niger contro la società

${ }^{30}$ O. De Schutter, 'Le contrôle du respect des droits de l'homme par les sociétés transnationales: le rôle de l'Etat d'origine', in M.-A. Moreau. H. Muir-Watt e P. Rodière (ed.), Justice et mondialisation en droit du travail, (Dalloz, 2010), 107, at 109.

${ }^{31}$ Kadic v. Karadzic, 70 F. 3d 232 (2d Cir. 1995).

${ }^{32}$ Doe I v. Islmaic Salvation Front, 993 F. SUpp. 3 (D.D.C. 1998).

${ }^{33}$ Hilao v. Estate of Marcos, 103 F.3d 789 (9th Cir. 1996); In re Estate of Marcos Human Rights Litigation (Hilao v. Marcos), 25 F.3d 1467 (9th Cir. 1994), cert. denied, 513 U.S. 1126 (1995); Trajano v. Marcos, 978 F.2d 493 (9th Cir. 1992), cert. denied, 508 U.S. 972 (1993); Si veda E.L. Lutz, 'The Marcos Human Righs Litigation: Can Justice Be Achieved in US Courts for Abuses that Occured Abroad?', (1994) 14 Boston College Third World Law Journal, 43; H.N. Ramy, 'In Re Restate of Ferdinand E. Marcos Human Rights Litigation: Alien Tort Claims Act, Official Torture as Violation of the Law of Nations under the Alien Tort Claims Act', (1994) 17 Suffolk Transnational Law Review, 578.

${ }^{34}$ Forti v. Suarez-Mason, 672 F. Supp. 1531 (N.D. Cal. 1987) on reconsideration 694 F. Supp. 707 (N.D. Cal. 1988); Quiros de Rapaport v. Suarez-Mason, Civ. No. 87-2057 (N.D. Cal. Apr. 22, 1988).

${ }^{35}$ John Doe I v. Unocal Corp., 963 F. Supp 880 (C.D. Cal. 1997).

${ }^{36}$ Forti v. Suarez-Mazon, 672 F. Supp. 1531 (N.D. Cal. 1987)

${ }^{37}$ B. Stephens, J. Chomsky, J. Green, P. Hoffman, M. Ratner, International Human Rights Litigation in U.S. Courts (2nd ed., Martinus Nijhoff Publishers, 2008) at 2.

${ }^{38}$ Kadic v. Karadzic, 70 F.3d 232 (2d Cir. 1995).

${ }^{39}$ Flores v. Southern Peru Copper Corp., 414 F.3d 233, Adbullahi v. Pfizer, Civ. No. 01-8118, 2002 U.S. Dist. LEXIS 17436 (S.D.N.Y. Sept. 16, 2002).

${ }^{40}$ Bowoto v. Chevron Texaco Corp., 312 F. Supp. 2d 1229 (N.D. Cal. 2004); Presbyterian Church of Sudan v. Talisman Energy, Inc., 453 F. Supp. 2d 633 (S.D.N.Y. 2006); Sarei v. Rio Tinto, PLC, Civ. Nos. 02-56256, PO-56390, 2007 WL 1079901 (9th Cir. Apr. 12, 2007).

${ }^{41}$ G. Peroni e C. Migani, op. cit. supra nota 6, p. 9.

${ }^{42}$ Kiobel v. Royal Dutch Petroleum Co., 621 F.3d 111, 125 (2d Cir. 2010).

${ }^{43} \mathrm{P}$. Acconci, La rilevanza per le imprese multinazionale degli obblighi convenzionali in materia di diritti della persona umana (Franco Angeli, 2011), at 299; D.M. Branson, 'Holding Multinational Corporations Accountable? Achilles' Heels in Alien Tort Claims Act Litigation', (2011) 9 Santa Clara Journal of International Law 227; M. Fasciglione, 'Il principio di corporate responsibility in materia di violazione dei diritti umani da parte di imprese multinazionali: il caso Kiobel', 
multinazionale Royal Dutch Shell. I promotori accusavano la società di essersi resa complice di violazioni dei diritti umani, chiedendo il supporto e sostenendo le forze armate Nigeriane per sopprimere le proteste non-violente della popolazione locale contro gli effetti dannosi dell'attività di estrazione petrolifera dell'impresa nella regione del Delta del Niger. Il punto di partenza del ragionamento della Corte federale di appello per il Secondo distretto è stato di rivolgersi al diritto internazionale, piuttosto che al diritto interno statunitense, per risolvere il problema della soggettività giuridica delle persone giuridiche nell'ambito del diritto internazionale. La Corte ha considerato che non si potesse dedurre dalla prassi internazionale, e più in particolare quella dei tribunali internazionali, che le imprese multinazionali, pur non essendo soggetti di diritto internazionale, possono essere responsabili di violazioni delle norme del diritto internazionale. Al contrario, altri tribunali hanno adottato la soluzione opposta. ${ }^{44}$ La Corte Suprema riformulò il problema di legge in un problema di legittimità dell'esercizio della giurisdizione extraterritoriale civile. ${ }^{45}$ La Corte Suprema considerò che l'esercizio della competenza universale civile non era legittimato nel caso in discussione alla luce della presunzione di esclusione dell'extraterritorialita ${ }^{46}$ prevista dal diritto americano. ${ }^{47} \mathrm{La}$ Corte ritenne che l'esistenzia di un ufficio direzionale sul territorio del foro non fosse un criterio di collegamento sufficente.. La Corte lasciò aperta il ricorso all'ATS nelle controvsersie non puramente extrateritoriali che entrengono alcuni collegamenti sufficientemente rilevante con il territorio americano. $^{48}$

(2011) 1 Diritti umani e diritto internazionale 131; C. Kearney, 'Corporate Liability Claims Not Actionable under the Alien Tort Statute - Kiobel v. Royal Dutch Petroleum Co.' (2011), 34 Suffolk Transnational Law Review 263; J.G. Ku, 'The Curious Case of Corporate Liability under the Alien Tort Statute: A Flawed System of Judicial Lawmaking', (2010) 51 Virginia Journal of International Law 353; M. Teophila, "Moral Monsters' Under the Bed: Holding Corporations Accountable for Violations of the Alien Tort Statute After Kiobel v. Royal Dutch Petroleum Co.', (2011) 79 Fordham Law Review 2859; N.C. Thompson, 'Putting the Cart Back Behind the Horse: The Future of Corporate Liability Under the Alien Tort Statute After Kiobel', (2011) 9 DePaul Business and Commercial Law Journal 293; E. Pistoia, 'La fine di una giurisprudenza? Sulla sentenza Kiobel v. Royal Dutch petroleum in tema di applicazione dell' Alien Tort Statute alle società' (2011) 94 Rivista di diritto internazionale 480; D.R. Tawill, 'Kiobel v. Royal Dutch Petroleum Co.: The Second Circuit Rejects Corporate Liability Under the Alien Tort Statute', (2011) 19 Tulane Journal of International and Comparative Law 709; M. Winkler, 'Kiobel v. Royal Dutch Shell, ovvero cronaca di una morte (quasi) annunciata: niente più azioni di risarcimento nei confronti di imprese multinazionali per violazioni dei diritti umani', (2011) Diritto del commercio internazionale 213; F.-J. Zamora Cabot, 'Kiobel v. Dutch Corp. y los litigios internacionales sobre derechos humanos', (2011) 4 Papeles el tiempo de los derechos 1; H. Muir-Watt, 'Les enjeux de l'affaire Kiobel : le chaînon manquant dans la mise en oeuvre de la responsabilité des entreprises multinationales en droit international public et privé', (2010-2012) Travaux du comité français de droit international privé 233.

${ }^{44}$ Flomo v. Firestone Natural Rubber Co., 643 F.3d 1013, 1021 (7th Cir. 2011); Doe VIII v. Exxon Mobil Corp., 654 F.3d 11; 2011 U.S. App. LEXIS 13934, *4 (D.C. Cir. 2011).

45 "whether and under what circumstances the Alien Tort Statute (...) allows courts to recognize a cause of action for violations of the law of nations occurring within the territory of a sovereign other than the United States."

${ }^{46}$ M.K. Fiechter, 'Extraterritorial Application of the Alien Tort Statute: The Effect of Morrison v. National Australia Bank, Ltd. on Future Litigation', (2012) 97 Iowa Law Review 959, at 968.

47 Kiobel v. Royal Dutch Petroleum Co., No. 10-1491 (U.S. Sup. Ct. Apr. 17, 2013). A. Bonfanti, 'No Extraterritorial Jurisdiction Under the Alien Tort Statute: Which Forum for Disputes on Corporate Human Rights Violations after Kiobel?', (2013) 2 Diritti Umani e Diritto Internazionale 377; V. Curran, 'Extraterritoriality, Universal Jurisdiction and the Challenge of Kiobel v. Royal Dutch Petroleum Co.,' (2013) 28 Maryland Journal of International Law 76; H. MuirWatt, 'L'Alien Tort Statute devant la Cour Suprême des Etats-Unis. Territorialité, diplomatie judiciaire ou économie politique?' (2013) 3 Revue critique de droit international privé 595; F.J. Zamora Cabot, 'Las empresas multinacionales y su responsabilidad en materia de derechos humanos: una vision de conjunto', (2013) 6 Papeles el tiempo de los derechos 1; G. Skinner, R. McCorquodale, O. De Schutter, and A. Lambe, The Third Pillar: Access to Judicial Remedies for Human Rights Violations by Transnational Business (December 2013): http://www.biicl.org/documents/182_the third_pillar.pdf; C. Bright, 'The Implications of the Kiobel v. Royal Dutch Petroleum Case for the Exercise of Extraterritorial Jurisdiction' in A. Di Stefano (ed.), A Lackland Law? Territory, Effectiveness and Jurisdiction in International and EU Law (Torino: Giappichelli) 165.

${ }^{48}$ La corte declarò: 'On these facts, all the relevant conduct took place outside the United States. And ever where the claims touch and concern the territory of the United States, they must do so with sufficient force to displace the presumption against extraterritorial application ... Corporations are often present in many countries, and it would reach too far to say 
Occorre comunque vagliare questa decisione alla luce del diritto internazionale, poiché altri Stati potrebbero esercitare la giurisdizione universale civile, in particolare nell'ordinamento europeo, con la recente proposta di estendere l'applicazione del regolamento Bruxelles I ai convenuti di paesi terzi (che anche se non è stata adottata nella recente revisione del regolamento, potrebbe vedere luce nel futuro).

A questo riguardo, il principio di universalità - che è uno dei titoli di giurisdizione riconosciuti in diritto internazionale per l'esercizio extraterritoriale della giurisdizione - potrebbe servire da base giuridica. In materia penale, è generalmente accettato che uno Stato possa esercitare la giurisdizione in modo extraterritoriale, a prescindere da qualunque collegamento tra crimine e lo Stato del foro, ${ }^{49}$ nei confronti di certi crimini tanto gravi da offendere valori comuni alla comunità internazionale. Tale esercizio della giurisdizione universale, autorizzato in diritto internazionale, è anche a volte reso obbligatorio. ${ }^{50}$ Diverse convenzioni internazionali impongono agli Stati l'obbligo di estradare oppure di perseguire, in conformità con il principio aut dedere aut iudicare, i presunti colpevoli di certi crimini quando essi si trovino sul loro territorio. Questi crimini includono i crimini di guerra, ${ }^{51}$ gli atti di tortura ${ }^{52}$ e le sparizioni forzate. ${ }^{53} \mathrm{Al}$ di là di queste obbligazioni di origine convenzionale, è stato

(Contd.)

that mere corporate presence suffices. If Congress were to determine otherwise, a statute more specific that the ATS would be required.'

${ }^{49}$ H. Ascensio, 'Are Spanish Courts Backing Down on Universality? The Supreme Tribunal's decision in Guatemalan Generals', (2003) 1 Journal of International Criminal Justice 690, at 699: "(universal jurisdiction) is usually defined negatively, as a ground of jurisdiction which does not require any link or nexus with the elected forum"; L. Reydams, Universal Jurisdiction. International and Municipal Legal Perspectives (Oxford University Press, 2003), at 5; R. O'Keefe, 'Universal Jurisdiction: Clarifying the basic concept', (2004) 2 Journal of international Crimintal Justice 735, at 745: "universal jurisdiction amounts to the assertion of jurisdiction to prescribe in the absence of any other accepted jurisdictional nexus at the time of the relevant conduct".

${ }^{50}$ G. Guillaume, "La compétence universelle. Formes anciennes et nouvelles", Mélanges offers à Georges Levasseur (Litec, 1992) 33; A. Cassese, 'Is the Bell Tolling for Universality; A plea for a sensible notion of universal jurisdiction', (2003) 1 Journal of International Criminal Justice 589; G. Abi-Saab, 'The Proper Role of Universal Jurisdiction', (2003) 1 Journal of International Criminal Justice 596; M. Henzelin, Le principe de l'universalité en droit pénal international: droit et obligation pour les Etats de poursuivre et juger selon le principe de l'universalité (Bruylant, 2000); L. Reydams, Universal Jurisdiction: International and Municipal (Perspectives, OUP, 2003).

${ }^{51}$ Convenzioni di Ginevra del 12 agosto 1949 (I) Convenzione di Ginevra per la protezione dei feriti e dei malati nelle forze armate di terra, art. 49; (II) la protezione dei feriti e dei malati nelle forze armate di terra, dell'aereonautica e della Marina, art. 50; (IIIe) relativa alla tutela dei prigionieri di guerra, art. 129; Convenzione di Ginevra per la protezione delle persone civili in tempo di guerra, art. 146: "Le Alte Parti contraenti s'impegnano a prendere ogni misura legislativa necessaria per stabilire sanzioni penali adeguate da applicarsi alle persone che abbiano commesso o dato ordine di commettere una delle infrazioni gravi alla presente Convenzione precisate nell'articolo seguente. Ogni parte contraente avrà l'obbligo di ricercare le persone imputate di aver commesso o di aver dato l'ordine di commettere una di dette infrazioni gravi e dovrà, qualunque sia la loro nazionalità, deferirle ai propri tribunali. Essa potrà pure, se preferisce $\mathrm{e}$ secondo le norme previste dalla propria legislazione, consegnarle, per essere giudicate, ad un'altra Parte contraente interessata al procedimento, purchè quessta parte contraente possa far valere contro dette persone prove sufficienti". L'articolo seguente precisa che le infrazioni gravi menzionati sono "l'omicidio intenzionale, la tortura o i trattamenti inumani, compresi gli esperimenti biologici, il fatto di cagionare intenzionalmente grandi sofferenze o di attentare gravemente all'integrità fisica o alla salute, la deportazione o il trasferimento illegali, la detenzione illegale, il fatto di costringere una persona protetta a prestar servizio nelle forze armate della Potenza nemica, o quello di privarla del suo diritto di essere giudicata regolarmente e imparzialmente secondo le prescrizioni della presente Convenzione, la presa di ostaggi, la distruzione e l'appropriazione di beni non giustificate da necessità militari e compiute in grandi proporzioni ricorrendo a mezzi illeciti e arbitrari".

${ }^{52}$ Convenzione contro la tortura ed altre pene o trattamenti crudeli, disumani o degradanti, adottata dall'Assemblea Generale delle Nazioni Unite il 10 dicembre 1984, ed entrata in vigore il 26 giugno 1987, art. 5.2: "Ogni Stato Parte prende (...) i provvedimenti necessari al fine di stabilire la propria competenza per conoscere dei suddetti reati qualora il presunto autore si trovi in un territorio sotto la sua giurisdizione e qualora il suddetto Stato non lo estradi, conformemente all'articolo 8, verso uno degli Stati di cui al paragrafo 1 del presente articolo".

${ }^{53}$ Convenzione internazionale per la protezione di tutte le persone dalla sparizione forzata del 2006, art. 9.2: "Ogni Stato Parte adotta (...) le misure che risultano necessarie per affermare la propria competenza ad esercitare la giurisdizione sui reati di sparizione forzata quando il presunto autore del fatto è presente su un territorio sotto la sua giurisdizione, salvo il 
sostenuto che il dovere di cooperazione degli Stati nella repressione dei più gravi crimini contemplati da norme di valore di jus cogens imporrebbe un obbligo simile di estradare oppure di perseguire tali crimini - anche se fossero stati commessi da stranieri contro stranieri - purché il presunto reo si trovasse sul territorio del foro. È sufficiente che l'imputato sia presente nello Stato del foro. Anche se queste disposizioni fanno riferimento alle persone fisiche, un ragionamento simile potrebbe permettere di imporre un obbligo, o quanto meno potrebbe autorizzare, gli Stati ad esercitare la giurisdizione universale sulle persone giuridiche come le imprese multinazionali presenti sul loro territorio.

Per altri diritti umani non contemplati da norme di valore di jus cogens, la giurisdizione universale civile potrebbe essere in conformità con le prescrizioni del caso Lotus, ${ }^{54}$ purché essa stia entro i limiti previsti dal diritto internazionale. Questi limiti derivano dai principi di uguaglianza sovrana delle nazioni e di non-ingerenza negli affari interni degli altri Stati. In particolare, per non costituire un'intrusione nella sfera di sovranità degli altri Stati, due condizioni sarebbero necessarie all'esercizio della giurisdizione universale civile (che sono le stesse che regolano l'esercizio della competenza universale in materia penale): 1) che il presunto reo sia presente sul territorio del foro il 2) che lo stato normalmente competente si astenga, o per incapacità o per mancanza di volontà, dal perseguire il presunto autore del crimine. ${ }^{55}$

\section{Conclusione}

In conclusione, quando lo Stato ospitante è incapace o riluttante ad ottemperare al proprio obbligo di proteggere i diritti umani delle persone che si trovano sul suo territorio, anche nei casi di abusi commessi da persone giuridiche, e di rimediare a tali abusi, altri Stati, come lo Stato di origine o, in alcune condizioni, Stati terzi, potrebbero avere un ruolo importante per garantire la protezione di diritti umani universalmente riconosciuti. In questa ottica, i meccanismi giurisdizionali di diritto internazionale privato che permettono l'esercizio della giurisdizione extraterritoriale civile si sono rivelati strumenti chiave per partecipare ad una migliore regolazione delle imprese multinazionali che operano nei Paesi in via di sviluppo. In particolare, i meccanismi in materia di azioni di risarcimento dei danni promosse dalle vittime dinanzi alle corti del Paese di origine dell'impresa o di Paesi terzi, hanno permesso di imporre obblighi e garantito, allo stesso tempo, ai ricorrenti di trovare un effettivo risarcimento per i danni subiti.

(Contd.)

caso che lo Stato estradi la persona o la consegni ad un altro Stato in conformità con i suoi obblighi internazionali o la consegni ad una corte penale internazionale di cui abbia riconosciuto la giurisdizione."

${ }^{54}$ Corte Permanente di giustizia internazionale, 7 settembre 1927, The case of the S.S. "Lotus", Francia c. Turchia, CPJI Publications, 1927, serie A, $\mathrm{n}^{\circ}$ 10, p. 19: "Far from laying down a general prohibition to the effect that States may not extend the application of their laws and the jurisdiction of their courts to personç, property'and acts outside their territory, it leaves them in this respect a wide measure of discretion which is only limited in certain cases by prohibitive rules; as regards other cases, every State remains free to adopt the principles which it regards as best and most suitable."

$55 \mathrm{C}$. Bright, L'accès à la justice civile en cas de violations des droits de l'homme par des entreprises multinationales (Florence: EUI, Cadmus, 2013), at 206: "l'exercice de la compétence civile universelle - calqué sur la compétence pénale universelle - serait, au minimum, légitime à l'égard des personnes physiques présentes sur le territoire du for. Par ailleurs, il serait également fondé à l'égard des entreprises multinationales présentes sur le territoire du for. En effet, le fondement régissant un tel exercice - à savoir la répression de crimes internationaux particulièrement répréhensibles aux yeux de la communauté internationale - est parfaitement applicable aux deux types de personnes. Ainsi, la nature particulière de certaines normes ayant valeur de jus cogens peut, dans une certaine limite, justifier des tempéraments à l'obligation de limitation de la compétence juridictionnelle. Le caractère de jus cogens des violations de droits de l'homme alléguées dans le cadre de l'affaire Kiobel aurait donc permis de légitimer, aux yeux du droit international public, la compétence civile universelle du for américain sur la base de l'ATS. Pour les autres types de droits de l'homme, qui ne constituent pas le jus cogens, un autre tempérament pourrait être trouvé dans le risque de déni de justice qui serait de nature à fonder l'exercice d'une compétence civile universelle." 

